\title{
Chirality and accurate structure models by exploiting dynamical effects in continuous-rotation 3D ED data \\ Keywords: absolute structure, dynamical scattering, continuous-rotation 3D ED
}

Authors:

Paul B. Klar ${ }^{1}$, Yaşar Krysiak ${ }^{1,2}$, Hongyi Xu ${ }^{3}$, Gwladys Steciuk ${ }^{1}$, Jung Cho ${ }^{3}$, Xiaodong Zou ${ }^{3}$, Lukas Palatinus ${ }^{1 *}$

\author{
Affiliations: \\ ${ }^{1}$ Institute of Physics of the Czech Academy of Sciences; Prague, Czech Republic \\ ${ }^{2}$ Institute of Inorganic Chemistry of the Leibniz University Hannover; Hannover, Germany \\ ${ }^{3}$ Department of Materials and Environmental Chemistry, Stockholm University; Stockholm, \\ Sweden \\ *Corresponding author. Email: palat@fzu.cz
}

\begin{abstract}
:
Dynamical diffraction effects are usually considered a nuisance for structure analysis from continuous-rotation 3D electron diffraction (3D ED) data like cRED and MicroED. Here we demonstrate that by accounting for these effects during the structure refinement, significantly improved models can be obtained in terms of accuracy and reliability with up to four-fold reduction of the noise level in difference Fourier maps in comparison to the standard structure determination routines that ignore dynamical diffraction. As dynamical diffraction effects break the inversion symmetry of the diffraction, they allow a quick, easy, and reliable determination of the absolute structure of chiral crystals.
\end{abstract}




\section{Introduction}

The knowledge of the atomic structure of a material is the basis for understanding and optimizing its properties. It is therefore no surprise that the most cited paper of the $21^{\text {st }}$ century describes a tool to solve and refine crystal structures from X-ray, neutron, and electron diffraction experiments (1). In the last decade, three-dimensional electron diffraction (3D ED) made huge technological and methodological steps forward (2-4) and is a rapidly growing method to determine the crystal structure of submicrometric crystals by means of transmission electron microscopy (TEM). 3D ED has proven its utility for all classes of crystalline materials, including metal-organic frameworks, zeolites, minerals, pharmaceutical compounds and proteins (5). Since 2019, continuous-rotation 3D ED is the predominant data acquisition mode (Fig. S1) which allows fast data collection at very low electron dose levels and has a simple experimental setup (Fig. S2) analogous to the standard rotation method (6) commonly used in X-ray diffraction (XRD) and neutron diffraction (ND).

Commonly applied structure refinement routines apply the kinematical approximation, which assumes that electrons are scattered by the crystal at most once and that the diffracted intensity of reflection $\mathbf{h}$ is proportional to the square of the structure-factor amplitude $\left(I_{\mathbf{h}} \sim\left|F_{\mathbf{h}}\right|^{2}\right)$. However, inelastic scattering, defects and especially multiple elastic scattering cause deviations from this proportionality, leading to high figures of merit and limited quality of the structure models (4). The dynamical theory of electron diffraction describing multiple scattering was established almost a century ago (7) and nowadays is implemented in many tools applying either multislice or the Bloch-wave approach to calculate, e.g., high-resolution (scanning) TEM images, convergent-beam electron diffraction patterns, and precession-assisted 3D ED (stepwise acquisition) intensities $(8,9)$. Basically, the theory describes how simultaneously excited beams interfere as they propagate through the crystal and predicts non-linear deviations from intensities 
expected by the kinematical approximation including non-zero intensities for systematically absent reflections, which is commonly observed in 3D ED experiments (10) (Fig. S3). These deviations depend on the crystal thickness, its orientation, and the structure factors of all interfering beams. A valuable side effect is the intrinsic sensitivity of the dynamical intensities to the absolute structure of non-centrosymmetric crystals (11). This geometric property is of highest relevance for, e.g., catalysis and pharmacology, because it is directly related to the absolute configuration of chiral molecules forming the crystal.

Despite several successful demonstrations (12-14), the analysis of dynamical effects in electron diffraction patterns is not yet considered a routine approach for the reliable determination of the absolute structure. Furthermore, the computation of integrated dynamical diffraction intensities has never been reported for continuous-rotation 3D ED data and therefore structure refinements on these data were limited by the kinematical theory of diffraction, which removes any information on the absolute structure.

In this work, we present a data reduction and dynamical refinement routine for the analysis of all commonly applied 3D ED measurement protocols (see Fig. S2 for their systematic overview). No modification of established experimental procedures is needed, and the method can be thus applied also to already existing experimental data. Based on both new and previously published 3D ED data sets of 12 organic and 6 inorganic compounds, we demonstrate the power of this method for routine structure analysis. We show the straightforward determination of the absolute structure based on experimental data from 49 crystals of 8 different chiral compounds. Furthermore, the method provides improved fits to the data, significantly improved accuracy of refined atomic positions, and large reduction of noise in the difference electrostatic potential maps allowing better distinction of fine structural features, such as hydrogen atoms. 


\section{Data reduction and refinement with virtual frames}

Experimental intensities in a single static electron diffraction pattern are very difficult to model due to their extreme sensitivity to effects like crystal thickness, mosaicity, bending, defects etc. (Fig. 1A). Therefore, refinements need to be based on integrated intensities determined by integration of the intensity profile as a function of the scattering angle, also referred to as rocking curve. In our method we need to preserve the information about simultaneously excited reflections. Therefore, the integration is achieved by summing experimental diffraction patterns into a set of so-called overlapping virtual frames (OVF). Each OVF is characterized by its average goniometer angle $\alpha_{\mathrm{v}}$ and the angular range $\Delta \alpha_{\mathrm{v}}$ covered by the virtual frame (Fig. 1B). Dynamical calculations based on the structure model are then performed for dozens of crystal orientations covering the angular range of the $\mathrm{OVF}$, resulting in idealized rocking curves for all reflections assigned to the OVF. Numerical integration of these rocking curves then yields the model integrated intensities $I_{\text {calc }}$ for each OVF. However, for reflections that are in exact diffraction condition close to one of the limiting Ewald spheres only incomplete rocking curves are calculated. Such partial intensities should not be used in the refinement and geometric filters are used to exclude them (15). Only integrated intensities of reflections with a minimum distance $D_{\mathrm{Sg}}$ from the limiting Ewald spheres and with a small ratio $R_{\mathrm{Sg}}=\left|S_{\mathrm{g}}\right| /\left(D_{\mathrm{Sg}}+\left|S_{\mathrm{g}}\right|\right)$ are considered in the refinement, where $S_{\mathrm{g}}$ is the excitation error of a reflection relative to the average goniometer position of the respective OVF (Fig. 1B). To include in the refinement as many measured reflections as possible, the $\alpha$ offset between two consecutive OVFs must be smaller than $\Delta \alpha_{\mathrm{v}}$ so that there is an overlap $\Delta \alpha_{0}$ corresponding to at least one experimental frame between them (Fig. 1C, Tables S1 and S2). Typical values for $\Delta \alpha_{\mathrm{v}}$ used in this study are $1.5^{\circ}$ to $3^{\circ}$, achieved by combining between 2 and 50 experimental frames to one virtual frame, with an

overlap $\Delta \alpha_{0}$ between $0.5^{\circ}$ and $1^{\circ}$. Optimal values of $R_{\mathrm{Sg}}{ }^{\max }$ are typically between 0.5 and 0.8 , and 
appropriate values of $D_{\mathrm{Sg}}{ }^{\min }$ are between 0.0015 and $0.0030 \AA^{-1}$ (Fig. S4). The concept of virtual frames ensures that both experimental and calculated intensities are properly integrated (Fig. S5). The least-squares refinement of a structural model is then performed in the usual way by minimising the weighted difference between $I_{\text {calc }}$ and $I_{\mathrm{obs}}(15,16)$. 

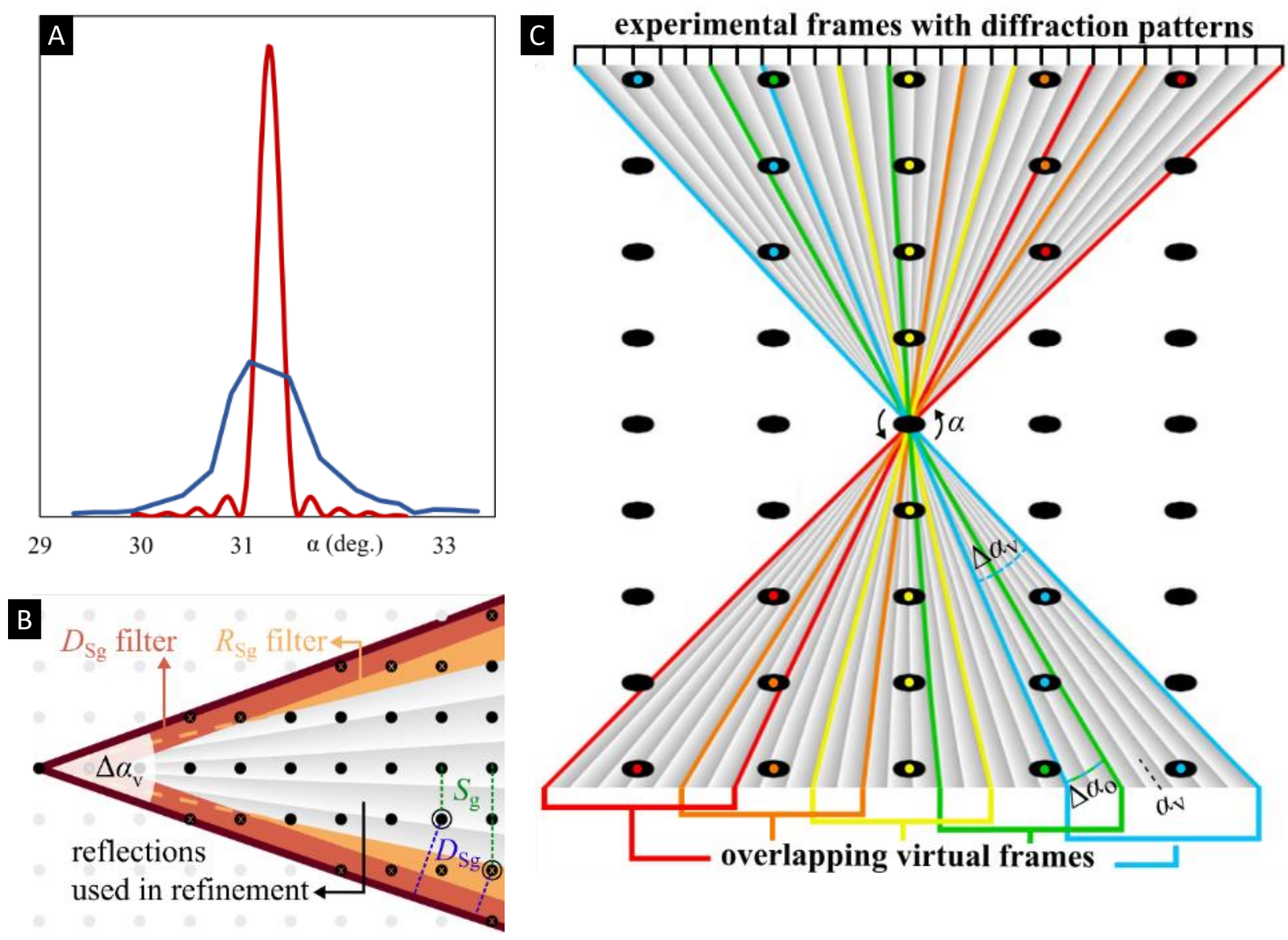

Fig. 1. Virtual frames in reciprocal space for continuous-rotation 3D ED. (A) Experimental (blue) and calculated (red) rocking curve of a reflection. Though experimental and calculated rocking curves differ substantially due to the crystal mosaicity, integrated intensities are in very good agreement. (B) Effect of $D_{\mathrm{Sg}}$ (red areas) and $R_{\mathrm{Sg}}$ (yellow areas) filters on selection of reflections (black disks) assigned to a virtual frame that are used in the dynamical refinement. Excitation error $S_{\mathrm{g}}$ and related $D_{\mathrm{Sg}}$ are drawn for two selected reflections (encircled). (C) Combination of experimental frames into overlapping virtual frames. In this illustration, seven frames contribute to one virtual frame, which overlap by two experimental frames. Reflections are marked by a dot with the colour of the corresponding virtual frame to which the reflection is assigned. 


\section{Improved models with dynamical refinement}

To evaluate the performance of the method, we analysed in detail the crystal structures of $\alpha$ quartz, albite, the zeolite structures mordenite, natrolite and STW_HPM-1, the framework compound cobalt aluminophosphate (CAP), the metal-organic framework CAU-36, in situ crystallised $\alpha$-glycine, the pharmaceutical compounds carbamazepine, (+)-limaspermidine and in situ crystallised abiraterone acetate as well as the methylene blue derivative MBBF4 (Table 1). We compare the results with the kinematical refinements performed on the same data. To ensure a fair comparison, we benchmarked our kinematical results against refinements obtained with other established software packages (Table S3).

We also compared the present method with dynamical refinements against precession-assisted 3D ED data of $\alpha$-quartz and natrolite. The comparison shows that obtained structural models are of similar quality. However, detailed analysis indicates that continuous-rotation 3D ED is more suitable for beam-sensitive materials and requires shorter computing times than precessionassisted 3D ED (see SI, Fig. S7, Tab. S4).

As the data processing is different for dynamical and kinematical refinement, we also made sure that the observed improvements are indeed due to the inclusion of the dynamical refinement and not because of the differences in data processing. We developed a method, named frame-based kinematical refinement (see SI for details), for performing the dynamical and kinematical refinements on exactly the same data. The comparison of the results shows that the observed improvement can indeed be predominantly attributed to the better description of dynamical effects (see SI, Fig. S8, Tab. S5).

In the following sections we illustrate the superiority of the dynamical refinement approach over the kinematical approach by comparing agreement factors ( $R$-factors), the overall accuracy of bond lengths in the refined models and the noise level in Fourier maps (Fig. 2, Tables S6-S17). 
Furthermore, we demonstrate that the dynamical refinement allows a straightforward determination of the absolute structure, which is absolutely impossible with kinematical refinement.

The most apparent improvement of applying dynamical refinement is the reduction of the $R$ factors (typically by a factor around two). Within the kinematical approximation, these residual factors are commonly calculated against symmetry-averaged reflection intensities, which are determined before the refinement. As the dynamical refinement does not include any prior averaging or merging of reflections, the refinement proceeds against non-averaged data. Since averaging of symmetry-equivalent reflections removes part of the noise in the data, the $R$-factors calculated on averaged data are lower than on non-averaged data. To allow for an unbiased comparison between the dynamical and kinematical refinement, we perform symmetry-averaging of experimental and calculated intensities and calculate the $R$-factors on these averaged values. We refer to these values as $M R$-factors. These $R$-factors are useful for presenting the quality of the fit, because they correspond best to the values crystallographers are used to. We evaluated the model accuracy using the root-mean-square deviation (RMSD) of refined covalent bond lengths from the respective reference values for all non-hydrogen atoms. The RMSD was in all cases lower for the dynamical refinement, on average by a factor of 1.5 (Fig. 2).

The correctness and completeness of a model is commonly inspected using difference Fourier maps of the electrostatic potential $\Delta V(\mathbf{r})$. In comparison to kinematical refinements, the dynamical refinement based on the same experimental data reduced the noise level of $\Delta V(\mathbf{r})$ by a factor of 1.5 to 4 (Fig. 2, Fig. 3, Tables S6-S17). Hence, weak structural features are more easily observed in difference maps obtained by dynamical refinement. This implies an easier detection of hydrogen atoms (see below). Furthermore, it allows a more reliable and precise location of 
guest molecules in zeolites and metal-organic frameworks (MOFs). We illustrate the improvement with two examples, a cobalt MOF CAU-36 (17) and a chiral zeolite STW_HPM-1 (18), which is a pure silica zeolite with STW topology, first discovered as germanosilicate SU-32 (19). In both cases, the dynamical refinement provided significantly improved difference electrostatic potential (DESP) maps (Fig. 3, Fig. S9). In STW_HPM-1 the dynamical refinement allowed an unambiguous detection of all individual atoms of the guest molecule, while the molecule was much less well defined in the kinematical result, with one half-occupied atomic position difficult to detect (Fig. 3B,C). In CAU-36, the dynamical DESP map allowed the location of a second orientation of the guest molecule, with the occupancy subsequently refined to $6,4 \%$ (SI, Fig. S9).

Another interesting case is the mineral albite. In a previous study using kinematical refinement, 16 data sets were combined to identify one correct out of 16 possible Al/Si distribution patterns exclusively from the $R$-factors (20). Using dynamical refinement, the reinvestigation of only 3 of the 16 data sets achieves a clearer identification of the correct element assignment from a more pronounced difference in $R$-factors between the candidate structures and from the analysis of the displacement parameters (Fig. S10 and S11). 


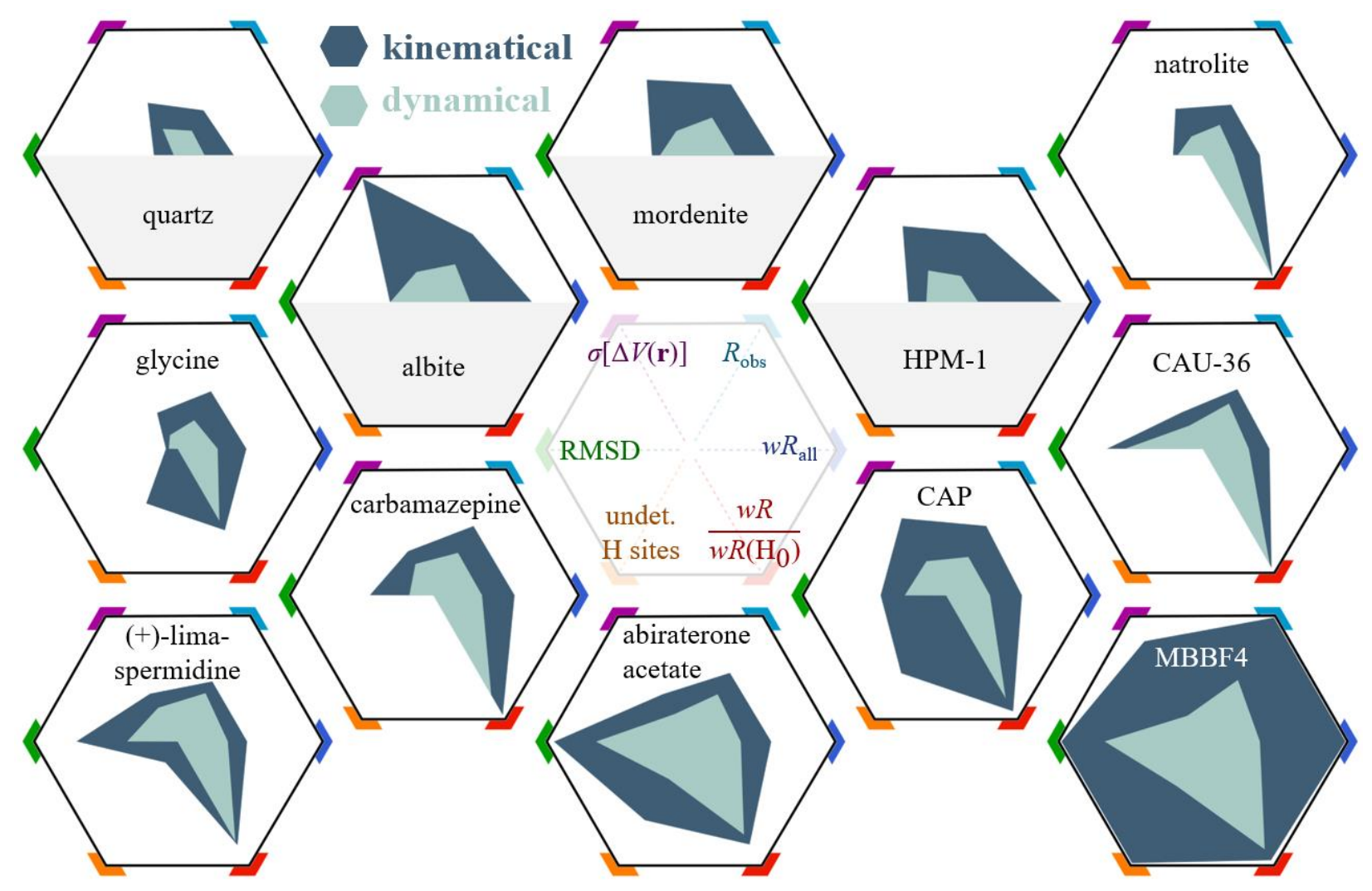

Fig. 2. Comparison of kinematical and dynamical refinements. Radar charts of key results of kinematical (dark blue, outer polygon) and dynamical (pale green, inner polygon) refinements. Smaller values close to the central white hexagon are better. $\sigma[\Delta V(\mathbf{r})]$ is the standard uncertainty of the difference Fourier map (purple, range: 0 to $0.372 \mathrm{e} / \AA$ ), $R_{\text {obs }}$ (light blue, range: 0 to 0.294 ) and $w R_{\text {all }}$ (dark blue, range: 0 to 0.333 ) are the respective $R$-factors, $R M S D$ is the root-meansquare deviation of the bond lengths (green, range: 0 to $0.603 \AA$ ), $w R_{\text {all }} / w R_{\text {all }}\left(\mathrm{H}_{0}\right)$ is the ratio of $w R_{\text {all }}$ of the refinement with and without hydrogen atoms (red, range: 0 to 0.997$)$, "undet. $\mathrm{H}$ sites" refers to the fraction of hydrogen atoms that could not be confirmed from difference Fourier maps (orange, range: 0 to 0.891 ). The latter two parameters are not applicable to quartz, albite, mordenite and STW_HPM-1. 
A
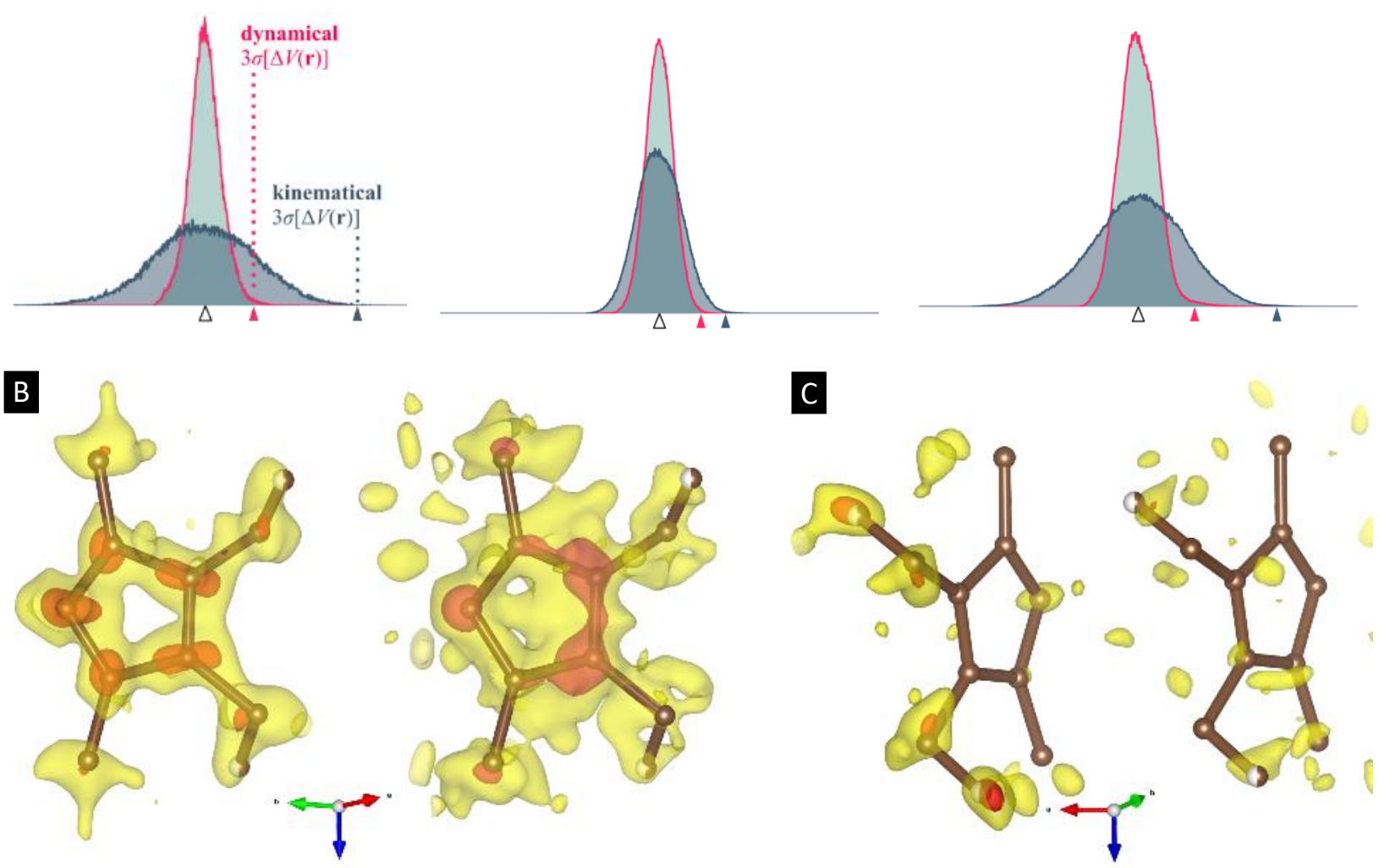

Fig. 3. Difference electrostatic potential maps. (A) Histogram of difference electrostatic potential maps $\Delta V(\mathbf{r})$ of selected kinematical (grey) and dynamical (pink) refinements. The bins (from left to right) that contain the values $\Delta V(\mathbf{r})=0 \mathrm{e} / \AA$ (black), $3 \sigma[\Delta V(\mathbf{r})]$ of the dynamical refinement (pink) and $3 \sigma[\Delta V(\mathbf{r})]$ of the kinematical refinement (blue) are marked on the horizontal axis. Each bin has a width of $0.0033 \mathrm{e} / \AA \AA$. (B), (C) DESP map from dynamical (left) and kinematical (right) refinement of STW_HPM-1 showing the guest molecule. (B): top view of the molecule with the DESP map obtained from the refinement without the guest molecule; $(\mathbf{C})$ : side view of the molecule with the DESP map obtained from the refinement with the guest molecule, but without the half-occupied terminal atom of the ethyl group. Isosurface levels are shown at $2.5 \sigma[\Delta V(\mathbf{r})]$ (yellow) and $4 \sigma[\Delta V(\mathbf{r})]$ (red). 


\begin{tabular}{llllrlll} 
Data source & Compound & Empirical formula & Space group & $V\left(\AA^{3}\right)$ & $T(\mathrm{~K})$ & Detector & $N_{\text {data }}$ \\
\hline this work & $\alpha$-quartz & $\mathrm{SiO}_{2}$ & $P 3{ }_{2} 21$ & 113.3 & 293 & $\mathrm{CCD}$ & 1 \\
$(20)$ & albite & $\mathrm{NaAlSi}_{3} \mathrm{O}_{8}$ & $P-1$ & 324 & 293 & $\mathrm{HPD}$ & 3 \\
$(21)$ & mordenite & $\mathrm{SiO}_{2}$ & $C m c m$ & 2850.4 & 293 & $\mathrm{HPD}$ & 1 \\
this work & natrolite & $\mathrm{Na}_{2} \mathrm{Al}_{2} \mathrm{Si}_{3} \mathrm{O}_{10}\left(\mathrm{H}_{2} \mathrm{O}\right)_{2}$ & $F d d 2$ & 2251.7 & 293 & $\mathrm{CCD}$ & 1 \\
this work & STW_HPM-1 & $\left(\mathrm{C}_{8} \mathrm{~N}_{2} \mathrm{H}_{15}\right) \mathrm{F} \mid\left[\mathrm{Si}_{10} \mathrm{O}_{20}\right]$ & $P 6_{1} 22$ & 3642.6 & 100 & $\mathrm{CMOS}$ & $1 *$ \\
this work & CAP & $\mathrm{C}_{1.14} \mathrm{Al}_{2} \mathrm{P}_{4} \mathrm{O}_{20} \mathrm{H}_{10.72}$ & $P 2{ }_{1} / n$ & 712.0 & 100 & $\mathrm{CCD}$ & 3 \\
$(17)$ & CAU-36 & $\mathrm{C}_{56} \mathrm{H}_{48} \mathrm{Co}_{2} \mathrm{~N}_{8} \mathrm{NiO}_{18} \mathrm{P}_{4}$ & $P-42 c$ & 4214.8 & 100 & $\mathrm{HPD}$ & 4 \\
$(22)$ & $\alpha$-glycine & $\mathrm{C}_{2} \mathrm{H}_{5} \mathrm{NO}_{2}$ & $P 2{ }_{1} / n$ & 304.7 & 100 & $\mathrm{HPD}$ & 1 \\
$(23)$ & carbamazepine & $\mathrm{C}_{15} \mathrm{H}_{12} \mathrm{~N}_{2} \mathrm{O}$ & $P{ }_{1} / n$ & 1144.8 & 100 & $\mathrm{CMOS}$ & 2 \\
$(23)$ & $(+)-$ limaspermidine & $\mathrm{C}_{19} \mathrm{H}_{26} \mathrm{~N}_{2} \mathrm{O}$ & $P 2_{12}{ }_{12}$ & 1594.4 & 100 & $\mathrm{CMOS}$ & 2 \\
this work & abiraterone acetate & $\mathrm{C}_{26} \mathrm{H}_{33} \mathrm{NO}_{2}$ & $P 2_{12}{ }_{21}$ & 2186.0 & 100 & $\mathrm{CCD}$ & 5 \\
$(24)$ & MBBF4 & $\mathrm{C}_{90} \mathrm{H}_{91} \mathrm{~B}_{4} \mathrm{~F}_{16} \mathrm{~N}_{21} \mathrm{~S}_{3}$ & $C 2 / c$ & 8973.0 & 293 & HPD & 8
\end{tabular}

Table 1. Sample overview. $V$ is the unit cell volume, $N_{\text {data }}$ is the number of measured data sets. Used abbreviations for detector types stand for charge-coupled device (CCD), hybrid pixel detector (HPD, also known as "direct electron detector") and complementary metal-oxidesemiconductor (CMOS). CAP samples were measured with static 3D ED, all other compounds with continuous-rotation 3D ED. *Another sample of STW_HPM-1 was measured and analysed independently at $T=293 \mathrm{~K}$ (HPD detector) as a blind test for the absolute structure determination. 


\section{Hydrogen atoms}

The low noise level is also a prerequisite for the successful detection of the weakest scatterers

(Fig. 4). Although the observation of hydrogen atoms in difference Fourier maps has been reported from kinematical refinements, it is by no means routine (Fig. S12 to S15). An illustrative example is the disordered framework structure CAP with partially occupied $\mathrm{Co}$ and $\mathrm{H}$ sites. Still the difference ESP map from dynamical refinement revealed all seven hydrogen positions with occupancies ranging from 0.5 to 1.0 as maxima larger than $4 \sigma[\Delta V(\mathbf{r})]$. Another hydrogen site with an expected occupancy of about 0.28 is detected close to the $3 \sigma$-level (See Si for details). Likewise, hydrogen atoms were easily detected in the organic compounds. All the 5, 12 and 26 hydrogen sites were clearly detected above $3 \sigma[\Delta V(\mathbf{r})]$ in the dynamical refinements of $\alpha$-glycine, carbamazepine and (+)-limaspermidine, respectively (Fig. 4d-f). In the cases of the more complex structures of abiraterone acetate and methylene blue derivative (MBBF4), still most $\mathrm{H}$ atoms were found (Fig. 2).

The accuracy and sensitivity of the dynamical refinement provides the means to investigate the refined lengths of covalent bonds involving hydrogen atoms in a quantitative way. In the young history of 3D ED, the constraints on hydrogen atoms in the refinement were mostly based on bond length statistics derived either from XRD or ND (Fig. S17). Even though the electrostatic potential is dominated by the positively charged nuclei, chemical bonds, which on average shift the negative potential of an electron, cause a shift of the observed overall potential away from that electron. Thus, the hydrogen atom position refined using the model of an independent neutral hydrogen atom is expected to be different from both the XRD-based distance, which is governed solely by the electron density, and the ND-based distance given by the position of the proton. Recently, this was briefly indicated in a cryo-EM single-particle study (25), but a quantitative evaluation of this effect has not yet been done. We performed a series of dynamical 
refinements varying the constrained $\mathrm{C}-\mathrm{H}, \mathrm{N}-\mathrm{H}$ and $\mathrm{O}-\mathrm{H}$ distances and compared the corresponding $w R_{\text {all }}$ (Fig. $4 \mathrm{~g}$ ). At $T=100 \mathrm{~K}$, the $\mathrm{C}-\mathrm{H}$ and $\mathrm{N}-\mathrm{H}$ distances in $\alpha$-glycine, carbamazepine and (+)-limaspermidine are less than $0.03 \AA$ longer than the respective internuclear distances. This difference is in excellent agreement with a theoretical analysis of the observed bond lengths within the independent atom model (cf. Fig. 13 in reference (26)). At room temperature, the $\mathrm{C}-\mathrm{H}$ distances in MBBF4 are longer by $0.05 \AA$. The $\mathrm{O}-\mathrm{H}$ distances in CAP $(T=100 \mathrm{~K})$ are longer by about $0.09 \AA$ and in natrolite $(T=293 \mathrm{~K})$ by $0.15 \AA$. The refinements thus reveal a clear trend towards longer refined bond lengths with a deviation that increases with the polarity of the covalent bond and the temperature. This observation calls for an extended study on a larger number of structures to achieve better understanding of this effect. The accuracy and sensitivity of the dynamical refinement are necessary prerequisites for the success of such study. 

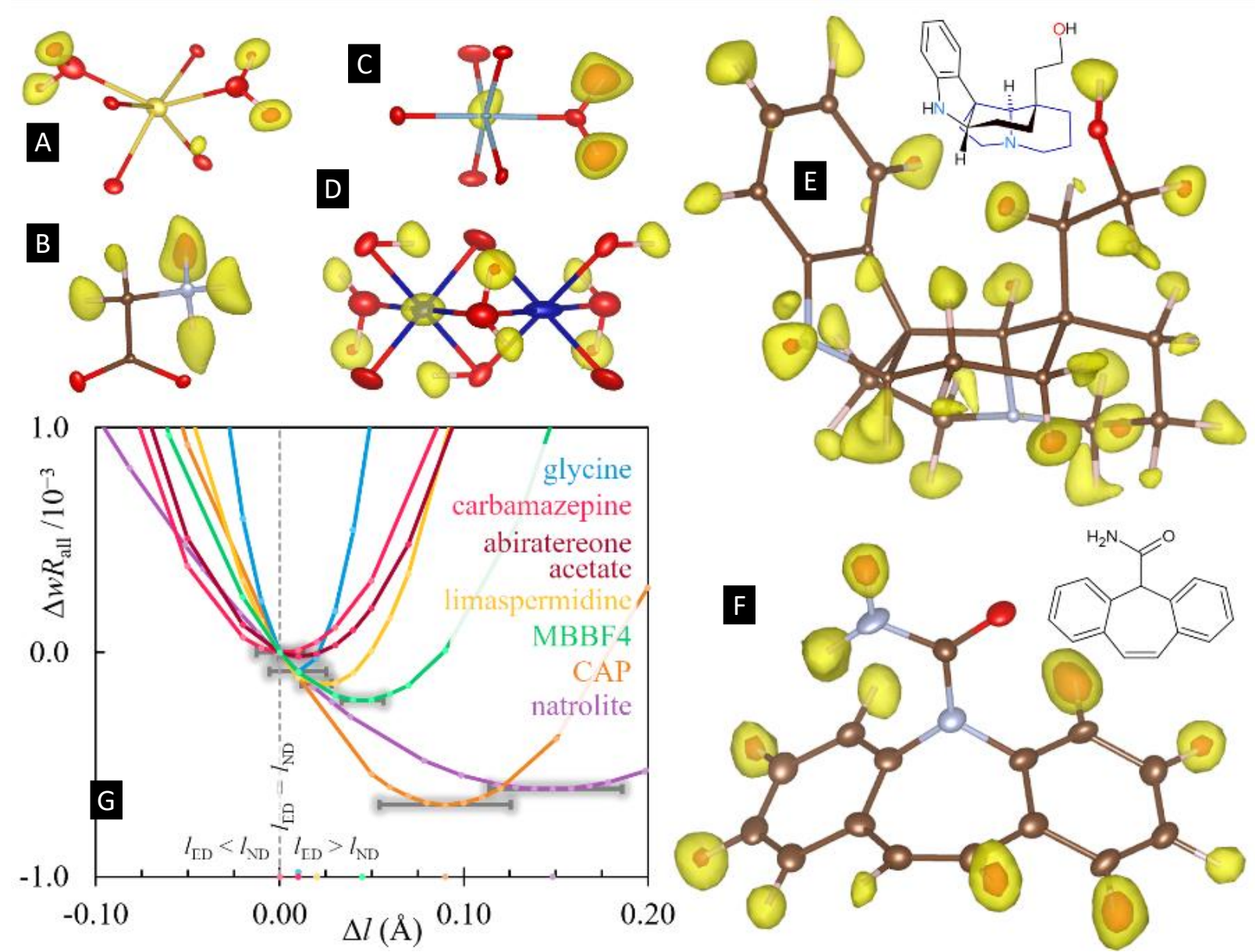

Fig. 4. Visibility and position of hydrogen atoms in electrostatic potential maps. Difference potential maps of natrolite (A), $\alpha$-glycine $(\mathbf{B}), \mathrm{CAP}(\mathbf{C}, \mathbf{D}),(+)$-limaspermidine $(\mathbf{E})$, and carbamazepine $(\mathbf{F})$ based on the final models with hydrogen atoms removed. Yellow isosurfaces correspond to the $3 \sigma[\Delta V(\mathbf{r})]$, red isosurfaces to the $5 \sigma[\Delta V(\mathbf{r})]$ level. The maps are superimposed with structure models with expected location of hydrogen sites indicated by lines from the respectively bonded $\mathrm{C}, \mathrm{N}$ or $\mathrm{O}$ atom. Anisotropic displacement ellipsoids shown at the $50 \%$ probability level. (G) Dependence of $w R_{\text {all }}$ on the $\mathrm{C}-\mathrm{H}, \mathrm{N}-\mathrm{H}$ and $\mathrm{O}-\mathrm{H}$ distances. $\Delta l$ is the difference between the constrained distances $l_{\mathrm{ED}}$ and the internuclear distance $l_{\mathrm{ND}}$ (27). Disks on the horizontal axis indicate the optimized difference of the apparent bond length that minimizes $w R_{\text {all. }}$ Horizontal error bars $(3 \sigma)$ are plotted at the optimal $\Delta l$ for each compound. 


\section{Determination of absolute structure}

Dynamical diffraction theory predicts a strong sensitivity of diffracted intensities to the absolute structure because of the interference of multiple excited beams. For example, the calculated intensities of (+)-limaspermidine and (-)-limaspermidine differ by $11.7 \%\left(1^{\text {st }}\right.$ quartile) to $61.1 \%$ $\left(3^{\text {rd }}\right.$ quartile) (Fig. S18). Even though inelastic scattering and crystal imperfections tend to diminish these differences, they still remain strong. As a blind test of the presented method, the chiral zeolite STW_HPM-1 was chosen as a suitable case study because during the synthesis relatively large single crystals grow as racemic conglomerate. The space group may be, depending on the chirality, either $P 6_{12} 22$ or $P 6_{5} 22$. A random sample was chosen for single crystal XRD measurement. Subsequently, the same crystal was crushed and prepared for continuous-rotation 3D ED (Fig. S19G). Dynamical refinements were performed of the two possible enantiomorphs converging to $w R_{\text {all }}$ of 0.306 for model in $P 6_{1} 22$ and 0.234 for $P 6_{5} 22$. The model in space group $P 6_{5} 22$ with the significantly lower $R$-factors was then confirmed as the correct enantiomorph by the determination of the Flack parameter of 0.06(2) from single-crystal XRD data (Tables S18 and S19).

As a demonstration of the robustness of the method and applicability to high-throughput routine determination of absolute structure, we investigated - in addition to (+)-limaspermidine and abiraterone acetate - all continuous-rotation 3D ED measurements of chiral non-protein structures publicly available in the 3DED/MicroED community on raw-data repository Zenodo (www.zenodo.org). Dynamical refinements of both enantiomorphs were carried out against individual data sets of (+)-limaspermidine ( 1 crystal), abiraterone acetate (5 crystals), (+)-biotin (20 crystals), progesterone ( 4 crystals), epicorazine B ( 4 crystals) and $\alpha, \beta$-dyhydrocurvularin ( 3 crystals), each with a completeness ranging from about $40 \%$ to $90 \%$ and a resolution in the range between 0.77 to $1.43 \AA$. Refinements against a combination of 6 data sets were performed for 
teniposide and an amyloid peptide. In all 49 cases, without exception, a simple comparison of the $R$-factors indicated the correct enantiomorph as the $w R_{\text {all }}$ of the model with wrong handedness is on average by a factor of 1.13 higher than the $w R_{\text {all }}$ of the correct model. In the cases of epicorazine B and $\alpha, \beta$-dyhydrocurvularin, the absolute configuration of the crystallised enantiomers derived from the dynamical refinements was again confirmed by X-ray diffraction by Novartis, who provided the samples. For other samples, their absolute structure was known in advance as they correspond to well characterized natural products. To quantitatively assess the reliability of the assignment of the enantiomorph, we adopted an approach inspired by le Page et al. (28) (see SI for details). The analysis provides both the confidence level in terms of $\sigma$ of the normal distribution ( $z$-score) as well as the probability that the estimation of the absolute structure is correct. The absolute structure assignments typically achieved a confidence level of $4.5 \sigma$ (median). Low-resolution data sets and split reflections in several cases reduced the confidence level, but in the worst case the probability for a correct enantiomorph assignment was $80.6 \%$ for one data set of teniposide, in all other cases it was above $95 \%$. More importantly, the dynamical refinement never favoured the wrong enantiomorph (Fig. 5, Tables S20 to S22). Recently, the potential of 3D ED for polymorph screening of in situ grown molecular crystals was investigated (22). With a similar approach, crystals of abiraterone acetate were grown directly on a TEM grid from an aqueous solution of abiraterone acetate citrate. Crystals diffracted up to a resolution of about $1 \AA$ at a temperature of $T=100 \mathrm{~K}$. Continuous-rotation 3D ED experiments were performed at different spots of selected crystals, which all showed signs of strong mosaicity (Fig. S19F). The identification of the correct enantiomorph was investigated at different stages of the structure determination for one of the data sets. The unrefined initial model based solely on the structure solution already identified the correct enantiomorph at the $3.5 \sigma$ confidence level. The subsequent refinement first without and then with constrained $\mathrm{H}$ 
atoms further increased the confidence level to $17.0 \sigma$ and $18.9 \sigma$ (Fig. 5B). Likewise, the absolute structure of (+)-limaspermidine was determined based on the unrefined model from the structure solution at $12.1 \sigma$ level (Fig. 5C). Note that the strongest scatterer of abiraterone acetate and (+)limaspermidine are $\mathrm{O}$ atoms. As the computational cost of the dynamical calculations scales with the number of refined parameters, the calculations for the initial absolute structure determination without refinement of structure model take only a few minutes with a standard personal computer (Table S4), making the determination of absolute structure in these cases very quick. In a further test, the robustness of the absolute structure determination of (+)-limaspermidine was investigated by gradually decreasing the number of OVFs used in the refinement, thus decreasing the data completeness. In the completeness range $100 \%$ to $25 \%$ the observed $R$-factor difference is almost constant around 0.05 , while the confidence level for the enantiomorph identification gradually decreases from $18.2 \sigma$ to $11.4 \sigma$. Even with a completeness of only $13 \%$, corresponding to an effective goniometer rotation of $9.2^{\circ}$, the refinement with constrained displacement parameters is stable and correctly identifies the enantiomorph at the $7.3 \sigma$ level (Fig. 5C, Tab. S23).

This analysis illustrates that 3D ED combined with dynamical refinement is a very reliable method for absolute structure determination. Furthermore, among the available methods to determine the handedness or chirality of single nanocrystals in a TEM $(12,13,29)$, the presented approach has to the best of our knowledge the least requirements in terms of TEM hardware, sample preparation and crystallite orientation as, e.g., no alignment of zone-axis patterns is needed and modern 3D ED setups measure full data sets in less only a few minutes $(24,30)$. With further automation, the presented approach is suitable for routine and high-throughput applications for all kinds of compounds without the need for a time-consuming and potentially expensive growth of larger single crystals. We argue that 3D ED may also be considered a 
valuable alternative to the established XRD-based approach even if single crystals of appropriate size are available, because the requirements for a successful determination in terms of crystal quality, measurement conditions, resolution, completeness, and elemental composition are strikingly reduced. This is especially relevant for pharmaceuticals, if the absolute structure determination by XRD is inconclusive or challenging $(31,32)$. However, if multiple limiting factors are combined, i.e., low completeness together with low-resolution and inferior crystal quality, the absolute structure determination by 3D ED may also yield a null result. 
$w R_{\text {all }}$ of correct $(\diamond)$ and wrong $(\square)$ enantiomorph

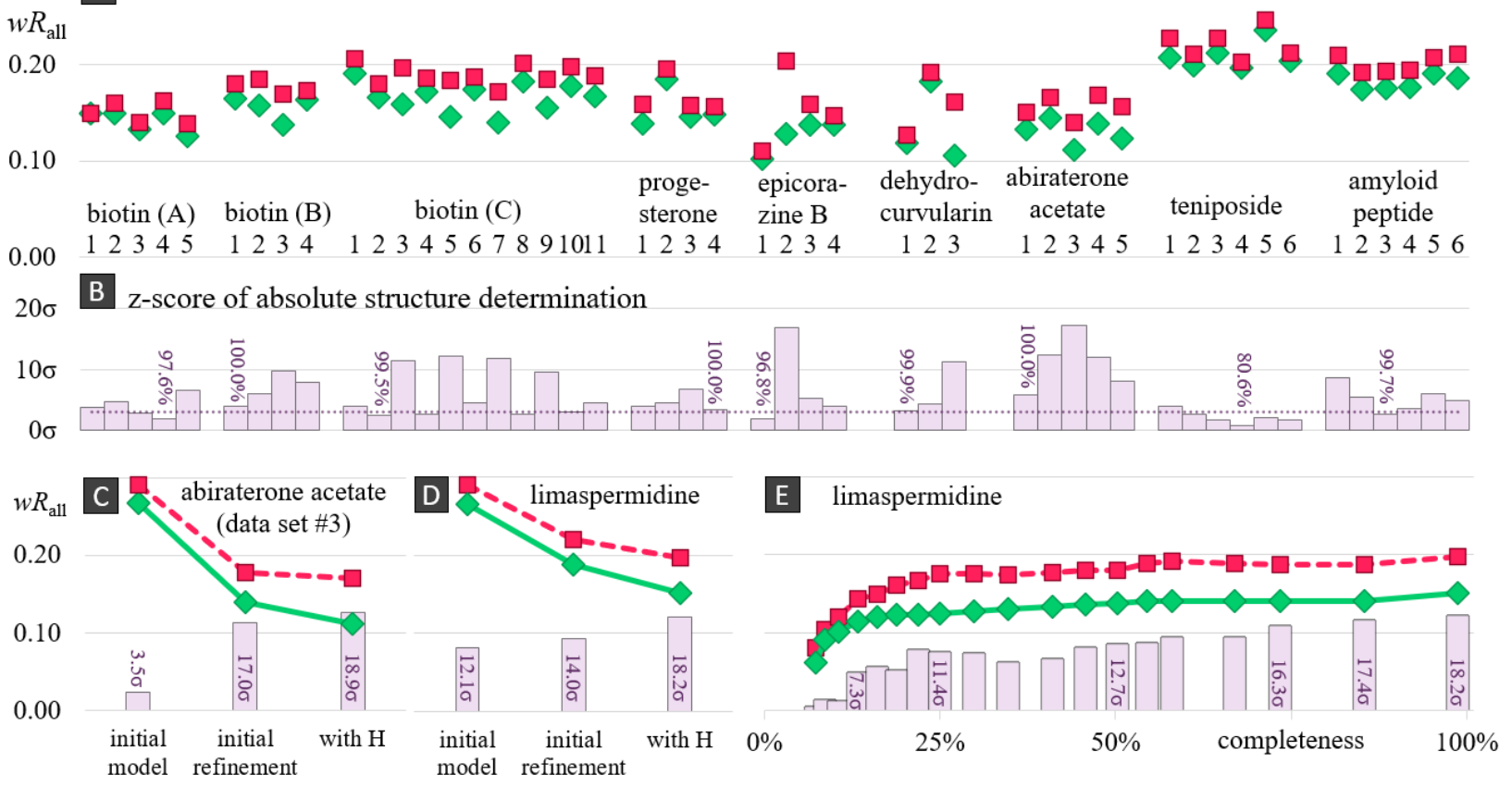

Fig. 5: Confidence and robustness of absolute structure determinations. (A) $w R_{\text {all }}$ of the dynamical refinement of the correct enantiomorph (green diamonds) and wrong enantiomorph (pink squares) of individual data sets. Non-hydrogen atoms were freely refined with isotropic displacement parameters. Hydrogen atoms were constrained with riding displacement parameter. (B) $z$-score expressing the confidence of the absolute structure assignment for each data set. For the weakest score in each set the corresponding probability for the correct assignment is given. The dotted line indicates the $3 \sigma$-limit, indicating a $99.7 \%$ probability. (C, D) $w R_{\text {all }}$ as a function of structure determination step for the enantiomorph pairs of abiraterone acetate and limaspermidine. The "initial models" are unrefined models based on the coordinates of the structure solution. The "initial refinement" refines coordinates and isotropic displacement parameters. "with $\mathrm{H}$ " includes constrained hydrogen atoms. The $\mathrm{z}$-score is represented by labelled vertical bars. (E) $w R_{\text {all }}$ as a function of the completeness of the set of reflections used in the refinements of the two enantiomorphs of limaspermidine. The z-score is represented by labelled vertical bars. 


\section{Conclusion and outlook}

A new data reduction and structure refinement procedure based on the concept of virtual frames was developed for static and continuous-rotation 3D ED data collection methods like ADT, RED, cRED and MicroED. Various structural features of a broad range of in total 18 compounds, including minerals, zeolites, molecular crystals, a MOF, several pharmaceutical compounds and an amyloid peptide, were studied applying the dynamical theory of electron diffraction. A comparison with the commonly applied kinematical refinement revealed that in all cases the dynamical refinement gives significantly improved results. Alongside better agreement factors, the benefits include an overall enhanced accuracy of atomic positions, decreased level of noise in the difference electrostatic potential maps and clearer detection of hydrogen atoms as well as guest molecules. The improved sensitivity of the analysis of $\mathrm{H}$ atoms showed that, within the independent atom model, the commonly applied constraint schemes in electron crystallography do not fit to the electron diffraction data. This observation must be considered for accurate modelling of $\mathrm{H}$ atoms.

In the case of non-centrosymmetric structures, the dynamical refinement easily identifies the correct enantiomorph by a simple comparison of corresponding $R$-factors, which is decidedly lower when refining the correct absolute structure. A thorough analysis of 49 data sets of molecular crystals with the correct identification of the known absolute structure establishes dynamical refinement and 3D ED as a reliable and generally applicable tool for the investigation of the absolute configuration of molecules forming submicrometric and nanosized crystals. The presented procedure for structure determination is compatible with all available 3D ED data collection methods. In total, 79 data sets from 10 different microscopes and from various laboratories were investigated. This work thus makes an accurate and deeper structure analysis accessible to a broad range of electron crystallography laboratories. As such this work allows to 
further close the gap in the quality and accessibility between 3D ED-based and XRD-based structure determinations. Nevertheless, this gap is evidently not fully closed yet, as can be seen from the still relatively high $R$-factors and related lower accuracy with respect to XRD results. However, we are certain that it is possible to narrow this gap even more by taking into account yet unmodelled aspects like inelastic scattering (33) or crystal imperfections. The dynamical refinement method presented in this work forms a basis for further investigation of these effects and for further improvement of the quality of structure analysis from 3D ED data.

\section{References and Notes}

1. G. M. Sheldrick, A short history of SHELX. Acta Cryst A. 64, 112-122 (2008).

2. E. Mugnaioli, T. Gorelik, U. Kolb, "Ab initio" structure solution from electron diffraction data obtained by a combination of automated diffraction tomography and precession technique. Ultramicroscopy. 109, 758-765 (2009).

3. L. Palatinus, P. Brazda, M. Jelinek, J. Hrda, G. Steciuk, M. Klementova, Specifics of the data processing of precession electron diffraction tomography data and their implementation in the program PETS2.0. Acta Crystallographica Section B-Structural Science Crystal Engineering and Materials. 75, 512-522 (2019).

4. T. Gruene, J. J. Holstein, G. H. Clever, B. Keppler, Establishing electron diffraction in chemical crystallography. Nat Rev Chem, 1-9 (2021).

5. M. Gemmi, E. Mugnaioli, T. E. Gorelik, U. Kolb, L. Palatinus, P. Boullay, S. Hovmoller, J. P. Abrahams, 3D Electron Diffraction: The Nanocrystallography Revolution. ACS Central Science. 5, 1315-1329 (2019).

6. Prince, E, International Tables for Crystallography Volume C: Mathematical, physical and chemical tables (International Union of Crystallography, ed. 3rd, 2006; https://it.iucr.org/C/).

7. H. Bethe, Theorie der Beugung von Elektronen an Kristallen. Annalen der Physik. 392, 55-129 (1928).

8. J. M. Zuo, J. C. H. Spence, Electron Microdiffraction (Springer US, 1992; https://www.springer.com/gp/book/9780306442629).

9. C. S. Own, L. D. Marks, W. Sinkler, Precession electron diffraction 1: multislice simulation. Acta Cryst A. 62, 434-443 (2006).

10. P. Oleynikov, S. Hovmöller, X. D. Zou, Precession electron diffraction: Observed and calculated intensities. Ultramicroscopy. 107, 523-533 (2007).

11. J. C. H. Spence, J. M. Zuo, M. O’Keeffe, K. Marthinsen, R. Hoier, On the minimum number of beams needed to distinguish enantiomorphs in X-ray and electron diffraction. Acta Cryst A. 50, 647-650 (1994).

12. H. Inui, A. Fujii, K. Tanaka, H. Sakamoto, K. Ishizuka, New electron diffraction method to identify the chirality of enantiomorphic crystals. Acta Cryst B. 59, 802-810 (2003).

13. Y. Ma, P. Oleynikov, O. Terasaki, Electron crystallography for determining the handedness of a chiral zeolite nanocrystal. Nature Materials. 16, 755-759 (2017). 
14. P. Brazda, L. Palatinus, M. Babor, Electron diffraction determines molecular absolute configuration in a pharmaceutical nanocrystal. Science. 364, 667-669 (2019).

15. L. Palatinus, V. Petříček, C. A. Corrêa, Structure refinement using precession electron diffraction tomography and dynamical diffraction: theory and implementation. Acta Crystallogr A Found Adv. 71, 235-244 (2015).

16. L. Palatinus, C. A. Corrêa, G. Steciuk, D. Jacob, P. Roussel, P. Boullay, M. Klementová, M. Gemmi, J. Kopeček, M. C. Domeneghetti, F. Cámara, V. Petříček, Structure refinement using precession electron diffraction tomography and dynamical diffraction: tests on experimental data. Acta Crystallogr B Struct Sci Cryst Eng Mater. 71, 740-751 (2015).

17. B. Wang, T. Rhauderwiek, A. K. Inge, H. Xu, T. Yang, Z. Huang, N. Stock, X. Zou, A Porous Cobalt Tetraphosphonate Metal-Organic Framework: Accurate Structure and Guest Molecule Location Determined by Continuous-Rotation Electron Diffraction. CHEMISTRY-A EUROPEAN JOURNAL. 24, 17429-17433 (2018).

18. A. Rojas, O. Arteaga, B. Kahr, M. A. Camblor, Synthesis, Structure, and Optical Activity of HPM-1, a Pure Silica Chiral Zeolite. J. Am. Chem. Soc. 135, 11975-11984 (2013).

19. L. Tang, L. Shi, C. Bonneau, J. Sun, H. Yue, A. Ojuva, B.-L. Lee, M. Kritikos, R. G. Bell, Z. Bacsik, J. Mink, X. Zou, A zeolite family with chiral and achiral structures built from the same building layer. Nature Mater. 7, 381-385 (2008).

20. E. Frojdh, J. T. C. Wennmacher, P. Rzepka, A. Mozzanica, S. Redford, B. Schmitt, J. A. van Bokhoven, T. Gruene, Discrimination of Aluminum from Silicon by Electron Crystallography with the JUNGFRAU Detector. CRYSTALS. 10 (2020), doi:10.3390/cryst10121148.

21. M. O. Cichocka, J. Angstrom, B. Wang, X. Zou, S. Smeets, High-throughput continuous rotation electron diffraction data acquisition via software automation. Journal of Applied Crystallography. 51, 1652-1661 (2018).

22. E. T. Broadhurst, H. Xu, M. T. B. Clabbers, M. Lightowler, F. Nudelman, X. Zou, S. Parsons, Polymorph evolution during crystal growth studied by 3D electron diffraction. IUCrJ. 7, 5-9 (2020).

23. C. G. Jones, M. W. Martynowycz, J. Hattne, T. J. Fulton, B. M. Stoltz, J. A. Rodriguez, H. M. Nelson, T. Gonen, The CryoEM Method MicroED as a Powerful Tool for Small Molecule Structure Determination. ACS Central Science. 4, 1587-1592 (2018).

24. T. Gruene, J. T. C. Wennmacher, C. Zaubitzer, J. J. Holstein, J. Heidler, A. Fecteau-Lefebvre, S. De Carlo, E. Mueller, K. N. Goldie, I. Regeni, T. Li, G. Santiso-Quinones, G. Steinfeld, S. Handschin, E. van Genderen, J. A. van Bokhoven, G. H. Clever, R. Pantelic, Rapid Structure Determination of Microcrystalline Molecular Compounds Using Electron Diffraction. Angewandte Chemie-International Edition. 57, 16313-16317 (2018).

25. T. Nakane, A. Kotecha, A. Sente, G. McMullan, S. Masiulis, P. M. G. E. Brown, I. T. Grigoras, L. Malinauskaite, T. Malinauskas, J. Miehling, T. Uchański, L. Yu, D. Karia, E. V. Pechnikova, E. de Jong, J. Keizer, M. Bischoff, J. McCormack, P. Tiemeijer, S. W. Hardwick, D. Y. Chirgadze, G. Murshudov, A. R. Aricescu, S. H. W. Scheres, Single-particle cryo-EM at atomic resolution. Nature. 587, 152-156 (2020).

26. B. Gruza, M. L. Chodkiewicz, J. Krzeszczakowska, P. M. Dominiak, Refinement of organic crystal structures with multipolar electron scattering factors. ACTA CRYSTALLOGRAPHICA A-FOUNDATION AND ADVANCES. 76, 92-109 (2020).

27. F. H. Allen, I. J. Bruno, Bond lengths in organic and metal-organic compounds revisited: $\mathrm{X}-\mathrm{H}$ bond lengths from neutron diffraction data. Acta Crystallographica Section B. 66, 380-386 (2010). 
28. Y. Le Page, E. J. Gabe, G. J. Gainsford, A robust alternative to $\eta$ refinement for assessing the hand of chiral compounds. J Appl Cryst. 23, 406-411 (1990).

29. Z. Dong, Y. Ma, Atomic-level handedness determination of chiral crystals using aberration-corrected scanning transmission electron microscopy. Nature Communications. 11, 1588 (2020).

30. M. Gemmi, M. G. I. La Placa, A. S. Galanis, E. F. Rauch, S. Nicolopoulos, Fast electron diffraction tomography. Journal of Applied Crystallography. 48, 718-727 (2015).

31. S. Parsons, H. D. Flack, T. Wagner, Use of intensity quotients and differences in absolute structure refinement. Acta Cryst B. 69, 249-259 (2013).

32. E. C. Escudero-Adán, J. Benet-Buchholz, P. Ballester, The use of Mo K $\alpha$ radiation in the assignment of the absolute configuration of light-atom molecules; the importance of high-resolution data. Acta Cryst B. 70, 660668 (2014).

33. T. Latychevskaia, J. P. Abrahams, Inelastic scattering and solvent scattering reduce dynamical diffraction in biological crystals. Acta Crystallographica Section B-Structural Science Crystal Engineering and Materials. 75, 523-531 (2019).

\section{Acknowledgments:}

We thank Tim Grüne, Julian Wennmacher, Jessica Bruhn, Christopher G. Jones, Hosea M.

Nelson, Emily Thompson, Huw Jenkins, Haruaki Yanagisawa and Keitaro Yamashita for sharing

their diffraction data and support with the data formats. Samples of quartz provided by Francesco Turci (University of Turin) and of abiraterone acetate provided by Martin Babor (Zentiva) are highly appreciated. We thank Yi Luo (Stockholm university) for checking the mordenite sample by IR spectroscopy. We acknowledge the assistance of Tim Grüne (University of Vienna), Jan Pieter Abrahams, Eric van Genderen (Paul Scherrer Institute) and Erik Maddox (Amsterdam Scientific Instruments) in understanding the confusion around the determination of absolute structure of epicorazine B and dehydrocurvularin. We are grateful to Trixie Wagner and Philippe Piechon (Novartis) for confirming the absolute structure of epicorazine B and dehydrocurvularin by single crystal X-ray diffraction.

\section{Funding:}

Czech Science Foundation, project number 19-08032S (PBK, YK, LP)

Czech Science Foundation, project number 21-05926X (PBK, LP) 
Operational Programme Research, Development and Education financed by European Structural and Investment Funds and the Czech Ministry of Education, Youth and Sports, Project No. SOLID21 CZ.02.1.01/0.0/0.0/16_019/0000760 (PBK, YK, LP)

MEYS CR, LM2018110 (CzechNanoLab Research Infrastructure) (PBK, YK, LP)

Knut and Alice Wallenberg Foundation, 2012.0112 and 2018.0237 (XZ)

Swedish Research Council, 2017-04321 (XZ)

Swedish Research Council, 2019-00815 (XZ)

Swedish Research Council, 2017-05333 (HX)

MicroED@SciLifeLab (HX)

Author contributions: (CRediT model)

Conceptualization: PBK, LP

Formal analysis: PBK, YK, HX, XZ, GS, LP

Investigation: PBK, YK, HX, GS, JC, XZ, LP

Methodology: PBK, LP

Resources: HX, XZ, LP

Software: PBK, YK, LP

Supervision: LP

Visualization: PBK, GS, LP

Writing - original draft: PBK, LP

Writing - review \& editing: PBK, YK, HX, GS, JC, XZ, LP

Data and materials availability: All used programs are available free of charge for academic purposes. The software PETS2 can be downloaded from pets.fzu.cz. The software package for structure solution and (dynamical) refinement with Jana2006/Jana2020, Dyngo and Superflip can be downloaded from jana.fzu.cz. Raw data (quartz, natrolite, CAP, abiraterone acetate, STW_HPM-1) and data reduction files are available at the data repository Zenodo (https://doi.org/10.5281/zenodo.5579792). 